\title{
Statistical scenarios forecasting method for wind power ramp events using modified neural networks
}

\author{
Mingjian CUI ( $₫)$, Deping KE, Di GAN, \\ Yuanzhang SUN
}

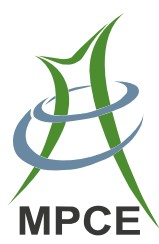

\begin{abstract}
Wind power ramp events increasingly affect the integration of wind power and cause more and more problems to the safety of power grid operation in recent years. Several forecasting techniques for wind power ramp events have been reported. In this paper, the statistical scenarios forecasting method is proposed for wind power ramp event probabilistic forecasting based on the probability generating model. Multi-objective fitness functions are established considering cumulative density functions and higher order moment autocorrelation functions with respect to the consistency of distribution and timing characteristics, respectively. Parameters of probability generating model are calculated by the iterative optimization using the modified genetic algorithm with multi-objective fitness functions. A number of statistical scenarios captured bands are generated accordingly. Eventually, ramp event probability characteristics are detected from scenarios captured bands to evaluate the ramp event forecasting method. A wind plant of Bonneville Power Administration with actual wind power data is selected for calculation and statistical analysis. It is shown that statistical results with multi-objective functions are more accurate than the results with single objective functions. Moreover, the statistical scenarios forecasting method can accurately estimate the characteristics of wind power ramp events. The results verify that the proposed method can guide the generation method of statistical scenarios and forecasting models for ramp events.
\end{abstract}

CrossCheck date: 5 January 2015

Received: 27 June 2014/ Accepted: 11 November 2014/Published online: 14 July 2015

(C) The Author(s) 2015. This article is published with open access at Springerlink.com

M. CUI, D. KE, D. GAN, Y. SUN, School of Electrical

Engineering, Wuhan University, Wuhan 430072, China

$(\bowtie)$ e-mail: mj_cui@whu.edu.cn
Keywords Neural networks, Genetic algorithm, Probability generating model, Statistical scenarios captured bands, Statistical scenarios forecasting, Wind power ramp events, Wind power

\section{Introduction}

Volatility, intermittency, randomness, and uncertainty of wind power can threaten the security and stability of power grid, and even cause serious losses [1]. Large fluctuations of wind power, such as significant increases or decreases in a short period, will form the so-called wind power 'ramp event' [2-4]. Wind power ramp events are particularly important in the management and the dispatch of wind power. It is necessary to regulate the output of traditional generators in power grid to make up for the substantial changes of wind power, such as using grid ancillary services or restricting and cutting down the ramp rate of wind turbine output. However, all the measures will cause major economic losses to the operation of power grid. The research of ramp event forecasting is an effective method to solve these issues which can help the power system operators, especially dispatching operators, developing wind power integration schemes and ancillary decisions in advance [5].

According to the difference of ramp directions, ramp events can be generally divided into two basic types: upramp and down-ramp [6]. The characteristic of the upramps is sharply increasing. Strong low pressure air systems (or cyclones), low-level jets, thunderstorms, wind gusts or similar atmospheric phenomena will induce upramps [7]. Likewise when the wind power suddenly drops or high speed gusts of wind make wind turbines reach cutout limits (usually $22 \sim 25 \mathrm{~m} / \mathrm{s}$ ), wind turbines would be shut down for protecting themselves from damages. All this phenomena could induce down-ramps [8]. Downramps are the reverse physical process of up-ramps [9]. 
Based on the property of each ramp event, ramp events are parameterized by the following variables: ramp start time, ramp duration, ramp rate, ramp swing, and ramp end time.

There are two major problems for forecasting wind power ramp events. As for the statistical or time-series models, the first problem is that the deterministic point prediction methods can obtain different results after simulations, such as the autoregressive moving average (ARMA) [10], neural networks (NNs) [11] and support vector machine (SVM) [12]. The second problem is the difficulty to forecast the ramp characteristics with respect to physical models [13]. These models use the surface roughness, orography, obstacles, atmosphere pressure, and temperature of a wind plant site, which is too complex to be simulated.

In this paper, a new wind power ramp event forecasting strategy is proposed and its efficiency is demonstrated by using several numerical experiments of an actual wind plant. The main contributions of this paper can be summarized as follows.

1) A novel and efficient forecast method for wind power ramp events is proposed. The forecast method is composed of a NN probability generating technique to generate forecast scenarios and a modified genetic algorithm to optimize model parameters. All the cumulative distribution features and higher order moment features of actual wind power can be considered to generate the forecast of wind power ramp events.

2) Less previous researches in the area of wind power ramp events focused on forecast methods. In this paper, an effective feature detecting technique, based on the forecasted statistical scenarios captured bands and the dynamic programming recursion algorithm, is used to detect all the forecasted ramp characteristics and compared with the actual ramp characteristics to verify the validity.

This paper is organized as follows. Section 2 reviews previous work on wind power ramp event forecast. Section 3 formulates the NN probability generating model and introduces a method to solve the optimal model parameters. Section 4 presents a ramp event detection algorithm. Section 5 presents the results of experiments in forecasting ramp events of an actual wind plant. Section 6 comes to the conclusion.

\section{Previous work}

The field of wind power ramp event detection and forecasting is a recent research hotspot in the sustainable energy sector. Especially as representatives, Raffi Sevilian from Stanford University and Nicholas Cutler from New South Wales University bringing this issue to a new research level. Sevilian defines a family of scoring functions with ramp event definitions and uses a dynamic programming recursion to detect all ramp events. It is also mentioned that the identified ramp information could be used in forecasting and simulation [14]. Cutler forecasts ramp events with the engineering application for the first time and evaluates the efficiency of the Wind Power Prediction Tool (WPPT) and the Mesoscale Limited Area Prediction System (MesoLAPS) for ramp event forecasting [15]. But sudden drops in an interval, determining a premature termination or start for ramp events cannot be excluded. Ramp events in [12] are grouped in classes and the SVM method is used to forecast and classify ramp events. This method maybe forecast ramp events successfully, but its lack of forecasting structure will make it undesirable for establishing models in future control applications, such as dispatching or unit commitment issues. Reference [16] used the ramp durations and ramp intensity of the predicted ramp events as additional variables to improve the reliabilities for the forecasted quantiles. It maps the number of ensemble members for forecasting a specific ramp event to a probability of that ramp actually occurring to produce confidence intervals of ramps occurring. But these confidence intervals of ramp events cannot be used conveniently without specific scenarios when referring to the dispatching or unit commitment issues. Reference [17] showed a user-friendly way of forecasting up-ramps and down-ramps with the uncertain start time and combines the numerical weather prediction (NWP) to bring the forecasting errors down. But for forecasting ramp events, better effect relies too much on better NWP forecasts. Better NWP forecast has a ramp capture nearly $10 \%$ higher than the combination and the other NWP forecasts.

\section{Problem formulation}

\subsection{NN probability generating model}

Assuming that the current moment is $t$, the input variables $X$ contains $(n+1)$ inputs, where $n$ inputs contain the current wind power (at time $t$ ) and historical wind power data (at time $t-1, t-2, \ldots, t-n+1$ ) and 1 input contains the possible wind power value of the next moment (at time $t+1)$. The output variable $O$ for the next moment contains the probability $p_{\tilde{X}_{t+1}, m}$ of the $m$ th possible wind power value. The NN probability generating model is shown in Fig. 1. 


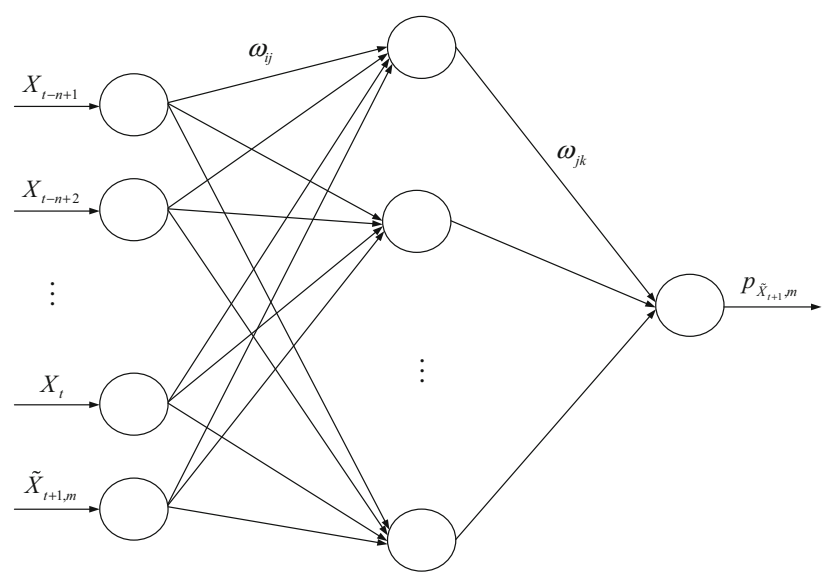

Fig. 1 NN probability generating model diagram

Due to the strongly nonlinear mapping relationship between the input and output variables, this model is established instead of the specific analytic expressions. Probability generating model has $(n+1)$ input layer nodes, 1 is the output layer node, and $l$ are hidden layer nodes, a total of $[(n+1) l+l \times 1]$ weight parameters and $(l+1)$ threshold parameters. Thus, there are $[(n+1) l+l \times 1+l+1]$ model parameters to solve. The calculation process of the model is as follows:

Step 1: Neural network initialization

The network input and output $(X \mid O)=\left(X_{t-n+1}\right.$, $\left.X_{t-n+2}, \ldots, X_{t-1}, X_{t}, \tilde{X}_{t+1, m} \mid p_{\tilde{X}_{t+1}, m}\right)$ needs to be determined. After initializing connection weight parameters $\left(\omega_{i j}\right.$ and $\omega_{j k}$ ) among the input, hidden and output layer and threshold parameters ( $\alpha$ and $\beta$ ) of the hidden layer and output layer, the learning rate and neuron excitation function are given.

Step 2: Hidden layer calculation

Hidden layer outputs $H$ are calculated based on the input variables $X$, connection weight parameters $\omega_{i j}$ and threshold parameters $\alpha$ as follows:

$h_{j}=f\left(\sum_{i=1}^{n+1} \omega_{i j} x_{i}-\alpha_{j}\right) \quad j=1,2, \ldots, l$

where $l$ is the hidden layer nodes; $f$ is the hidden layer excitation function.

There are many types of the excitation function formulations and this paper uses the function as:

$f(x)=\frac{1}{1+e^{-x}}$

Step 3: Output layer calculation

According to the hidden layer outputs $H$, connection weights $\omega_{j k}$ and threshold parameter $\beta$, the probability $p_{\tilde{X}_{t+1, m}}$ of the $m$ th possible wind power value at the next moment is calculated as the neural network output:
$p_{\tilde{X}_{t+1, m}}=\sum_{j=1}^{l} h_{j} \omega_{j k}-\beta \quad k=1$

$P_{\tilde{X}_{t+1}}=\left\{p_{\tilde{X}_{t+1,1}}, p_{\tilde{X}_{t+1,2}}, \ldots, p_{\tilde{X}_{t+1, m}}, \ldots, p_{\tilde{X}_{t+1, M}}\right\}$

$\sum_{m=1}^{M} p_{\tilde{X}_{t+1, m}}=1$

where $P_{\tilde{X}_{t+1}}$ is the totality of all the probability values; $M$ is the total number of possible wind power values at the next moment.

\subsection{Solving optimal model parameters}

The improved genetic algorithm is adopted to solve all the parameters of the NN probability generating model. One individual in the population represents all the weight and threshold parameters of one determinate neural network. Individual fitness value is calculated by the improved fitness function. And then the individual corresponding to the best fitness value is found by the selection, crossover and mutation operations. Eventually the optimal individual is created and assigned to the optimal weight and threshold parameters of the NN probability generating model.

\subsection{Multi-objective fitness function}

The core part of the improved genetic algorithm for solving model parameters is to establish a set of multiobjective function, which consists of the cumulative density function (CDF) and the higher order moment autocorrelation function. The absolute error $E_{1}$ is calculated between the forecasted and expected CDFs, and the absolute error $E_{2}$ is calculated between the forecasted and expected higher order moment autocorrelation functions. The final individual fitness $F$ is the sum of absolute errors $E_{1}$ and $E_{2}$ (with the equal weight) as:

$\min F=E_{1}\left(Y_{\mathrm{cdf}}, O_{\mathrm{cdf}}\right)+E_{2}\left(Y_{\text {corr }}, O_{\text {corr }}\right)$

$E_{1}=\sum\left|Y_{\mathrm{cdf}}-O_{\mathrm{cdf}}\right|$

$E_{2}=\sum\left|Y_{\text {corr }}-O_{\text {corr }}\right|$

where $Y_{\text {cdf }}$ is the expected CDF of mounts of actual historical wind power; $O_{\text {cdf }}$ is the forecasted CDF of the scenario sequence sampled by the NN probability generating model; $Y_{\text {corr }}$ is the expected higher order moment autocorrelation function value of mounts of actual historical wind power; $O_{\text {corr }}$ is the forecasted higher order moment autocorrelation function value of the scenario sequence sampled by the $\mathrm{NN}$ probability generating model. 


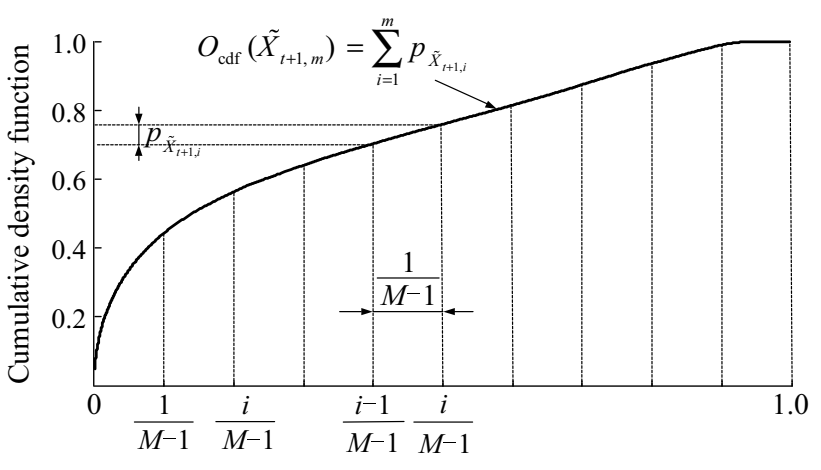

Wind power samples (p.u.)

(a) Probability of each sample point

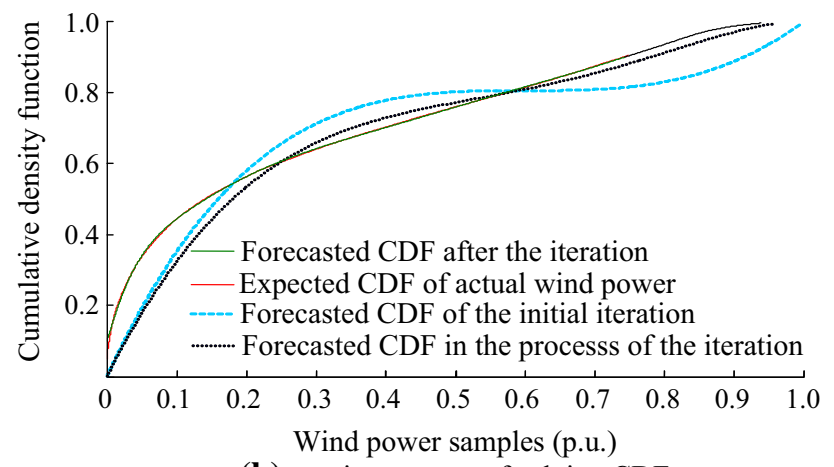

(b) terative process of solving CDF

Fig. 2 CDF generation and iterative schemes

\subsection{Cumulative density fitness function}

Based on the probability $p_{\tilde{X}_{t+1, m}}$ of the $m$ th possible wind power value and the definition of the cumulative density function, $\mathrm{CDF}$ can be calculated as:

$O_{\text {cdf }}\left(\tilde{X}_{t+1, m}\right)=\sum_{i=1}^{m} p_{\tilde{X}_{t+1, i}}$

After all the $M$ possible wind power forecasted value and their possibilities have been prepared, the method for generating the forecasted CDF will be performed. The horizontal axis is divided into $(M-1)$ equal and nonoverlapping intervals (with the width $1 /(M-1)$ of each interval). CDF generation and iterative schemes are shown in Fig. 2. The probability of each sample point has been generated by the $\mathrm{NN}$ probability generating model, as shown in Fig. 2a. The iterative process of solving CDF is shown in Fig. 2b. Equation (9) is modified as

$O_{\text {cdf }}\left(\tilde{X}_{t+1, m}\right)=O_{\text {cdf }}\left(\tilde{X}_{t+1, m-1}\right)+p_{\tilde{X}_{t+1, m}}$

\subsection{Higher order moment autocorrelation fitness} function

In fact, even if the forecasted and expected distribution of CDF is entirely fitting, the probability paper test result

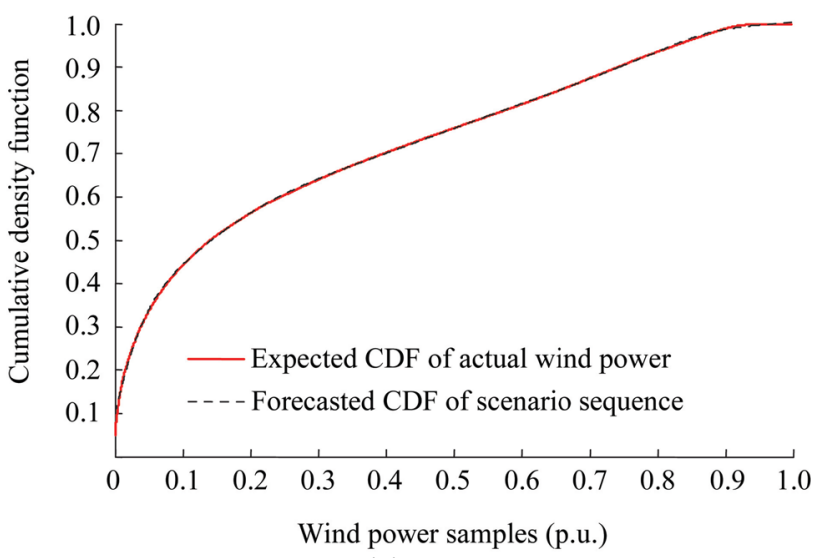

(a) CDF curves

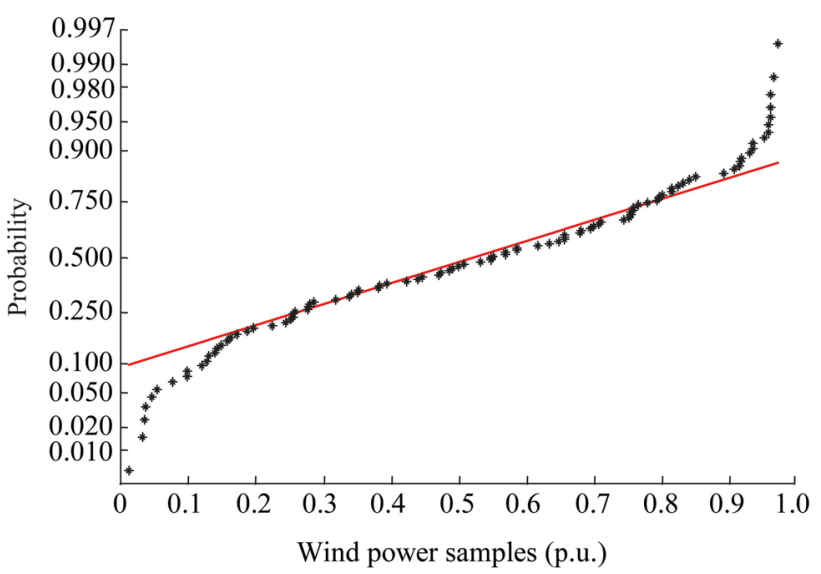

(b) Probability paper test

Fig. $3 \mathrm{CDF}$ curve and its probability paper test

shows tnat the distribution is just quasi [18], as shown in Fig. 3.

Figure 3a shows that the two CDFs fit completely, while the splashes in Fig. $3 \mathrm{~b}$ do not gather in a straight line. This phenomenon illustrates that the distribution of the forecasted CDF cannot obey that of the expected CDF. Even though the forecasted random sequence could satisfy the distribution properties (such as the first order moment statistical property of a random variable) of a certain actual wind power, the higher order moment [19] statistical properties are not consistent. Therefore, the higher order moment autocorrelation fitness function needs to be considered to establish the time sequence [18] between the forecasted and actual wind powers, and maintains the higher order moment features of the actual wind power sequence. The iterative process of the secondary order moment autocorrelation function is shown in Fig. 4.

\subsection{Population initialization}

Each individual is a real number string, that is, using real number coding method. Each individual consists of 


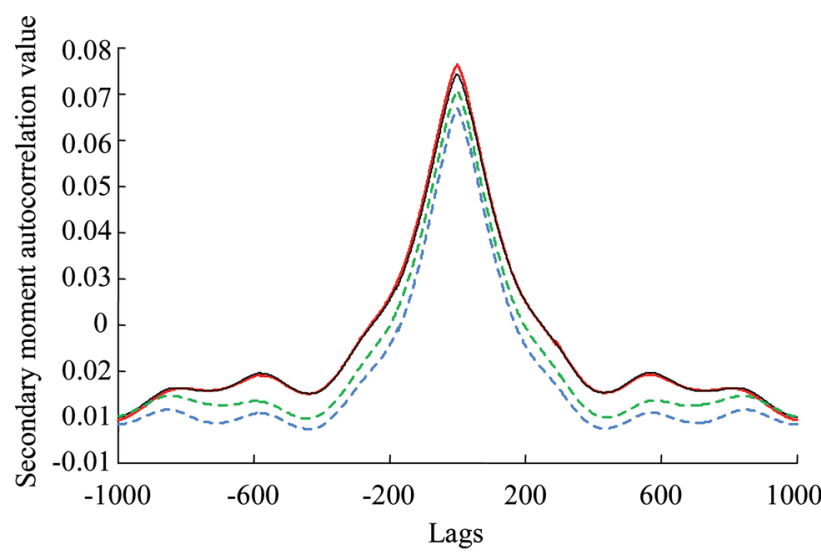

- Expected autocorrelation value of actual wind power

- Forecasted correlation value after the iteration

- - - Forecasted correlation value in the process of the iteration

- - - Forecasted correlation value of the initial iteration

Fig. 4 Iterative schemes of secondary order moment autocorrelation function

connection weights between the input layer and hidden layer, thresholds in the hidden layer, connection weights between the hidden layer and output layer, and the threshold in the output layer. Each individual contains all the NN weight and threshold parameters, and corresponds to a determinate $\mathrm{NN}$ probability generating model.

\section{Detecting algorithm for ramp events}

After solving optimal parameters of the forecasting model, forecasting scenarios and scenarios captured bands are sampled in quantity [20, 21]. Predicted characteristic values in all the scenarios are recognized by the detecting algorithm and the extensive statistical analysis is performed to calculate the probabilistic estimated values. The performance of the statistical scenarios forecasting model is evaluated by comparing the forecasted values with actual ones of ramp event characteristics.

Based on the large number of forecasting statistical scenarios, a latest detecting algorithm is adopted for the statistical analysis of prediction effects. The algorithm is proposed by Dr. Raffi Sevlian from Stanford University in 2012 [22]. It is also the first time that a set of the complete theoretical system for detecting ramp events is directly put forward (detecting effects of a certain period of the actual wind power, as shown in Fig. 5).

The detecting algorithm relies on the definition of a family of appropriate scoring functions. A dynamic programming recursive algorithm is used to identify and characterize all the ramp events needed for the power

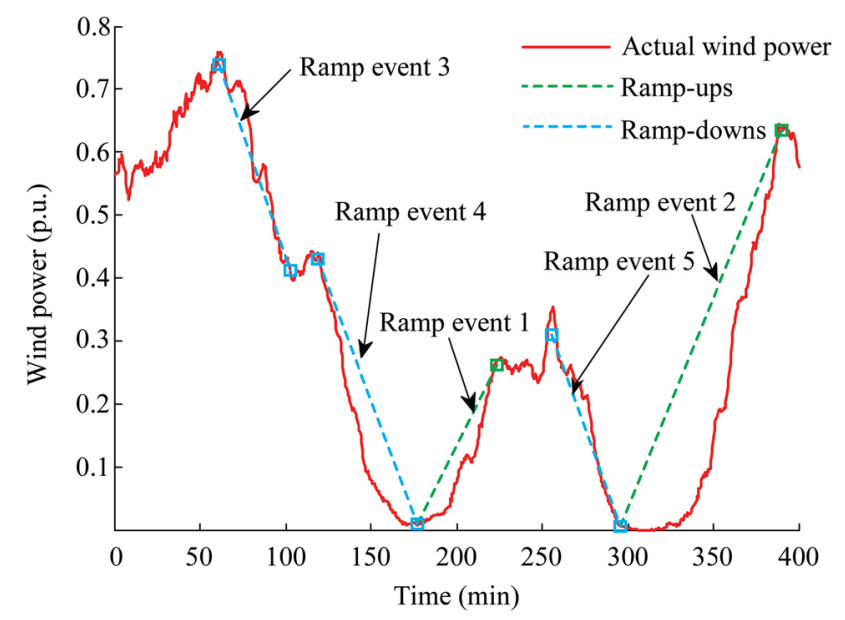

Fig. 5 Detected ramp events during a certain period

system operation, especially for the ancillary services and ramp event forecasting.

\section{Experimental results}

The actual wind power data is a time series from the Bonneville Power Administration (BPA). 184000 wind power samples are measured from the wind turbine output and sampled every $30 \mathrm{~s}$ with sampling time from January 1st, 2005 to December 31st, 2006. The selected wind plant capacity is $24 \mathrm{MW}$. It is noted that all the data are normalized on the basis of the rated capacity of this wind plant [23]. The experiments are executed on a desktop with i7$2640 \mathrm{M} \mathrm{CPU}$ at $2.80 \mathrm{GHz}$ with $3 \mathrm{~GB}$ RAM. To simplify the calculating process, $\mathrm{NN}$ probability generating model consists of 6 input layer nodes (five wind power values of historical moments and one possible wind power value of the next moment), 4 hidden layer nodes and 1 output layer node. The initial weight and threshold parameters are random numbers generated by MATLAB 2010a, and the optimal weight and threshold parameters are shown in Table 1.

\subsection{Forecasting results of statistical scenarios}

$D \%$ statistical scenarios captured band (SSCB) is defined to evaluate the validity of the forecasting model. The index definition refers to the ratio of $n_{\text {capt }}$ which is the number of actual wind power value falling into the SSCB and the total number of actual wind power data $n_{\mathrm{ttl}}$. When the actual wind power value falls into the SSCB, it means that the value is successfully captured and marked as 1 . Whereas when the actual wind power value falls outside the SSCB, it fails to be captured and marked as 0 . 
Table 1 Optimal weight and threshold parameters

\begin{tabular}{|c|c|c|c|c|c|c|}
\hline Parameters & Values & & & & & \\
\hline \multirow{4}{*}{$\omega_{i j}$} & -1.426 & 0.617 & -0.871 & -1.669 & -2.295 & -1.653 \\
\hline & -1.087 & -0.389 & -0.406 & -2.702 & -1.511 & 1.806 \\
\hline & -2.824 & 0.406 & 1.382 & -0.068 & 0.512 & -1.992 \\
\hline & -0.246 & 2.831 & 1.494 & 0.126 & -2.300 & 0.058 \\
\hline$\alpha$ & 0.7444 & 1.0748 & -0.626 & -0.795 & - & - \\
\hline$\omega_{j k}$ & 2.9296 & -2.7735 & 2.311 & -1.339 & - & - \\
\hline$\beta$ & 1.7771 & - & - & - & - & - \\
\hline
\end{tabular}

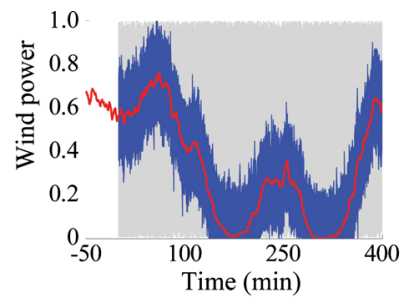

(a) $100 \%$ scenarios captured band

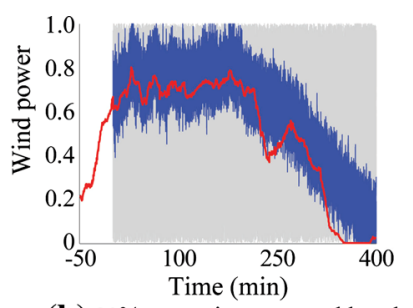

(b) $80 \%$ scenarios captured band

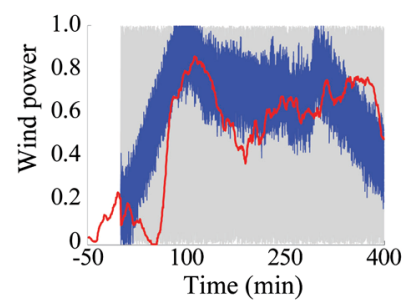

(c) $60 \%$ scenarios captured band

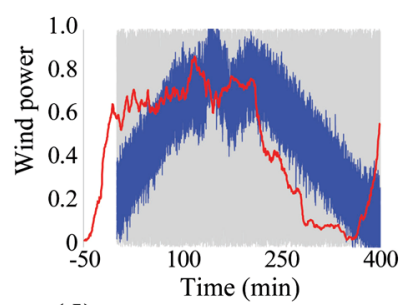

(d) $40 \%$ scenarios captured band

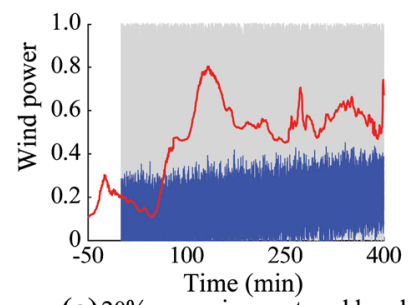

(e) $20 \%$ scenarios captured band

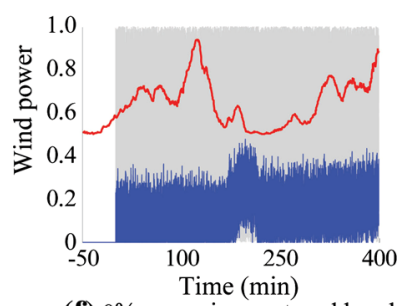

(f) $0 \%$ scenarios captured band

- Actural wind power; - Unsnapped scenarios; - SSCBs

Fig. 6 Prediction results of different statistical scenarios in $90 \%$ confidence interval

$D=\frac{\sum_{i=1}^{n_{\text {capt }}} 1}{n_{\mathrm{ttl}}} \times 100 \%$

Six SSCBs are taken an example for the quantitative description shown from Figs. 6, 7, 8 and 9. Different SSCBs forecasting actual wind power are respectively described with different confidence intervals $30 \%, 50 \%$, $70 \%$ and $90 \%$.

Meanwhile, in order to further verify the robustness of the statistical scenarios forecasting model, time window experiments are repeated ten thousand times. The statistical

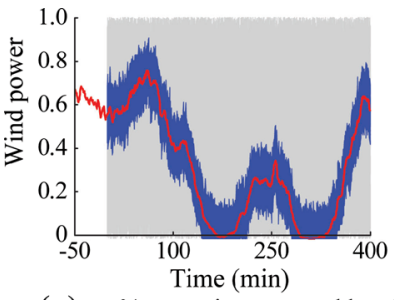

(a) $100 \%$ scenarios captured band

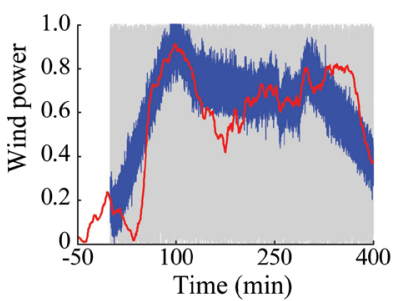

(c) $60 \%$ scenarios captured band

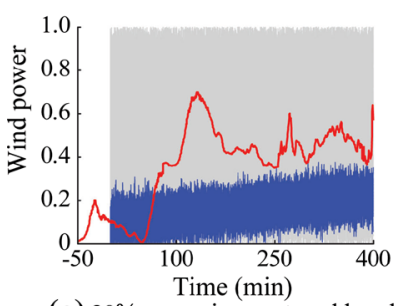

(e) $20 \%$ scenarios captured band

- Actural wind power; - Unsnapped scenarios; - SSCBs

Fig. 7 Prediction results of different statistical scenarios in $70 \%$ confidence interval

proportion of each SSCB occurring with different confidence intervals is shown in Fig. 10.

As shown from Figs. 6, 7, 8 and 9, different SSCBs in the same confidence interval can capture different actual wind power values. When the confidence interval is more than $50 \%, 100 \%$ SSCB with the highest percentage verifies the forecasting model with better effects. When the confidence interval equals to $30 \%, 90 \%$ SSCB occurs with the highest percentage. It illustrates that the percentage of $100 \%$ SSCB fully captures the actual wind power value occupies less and less with the shrinking of the confidence interval, which means that the confidence interval should not be too narrow in ramp event forecasting model. 


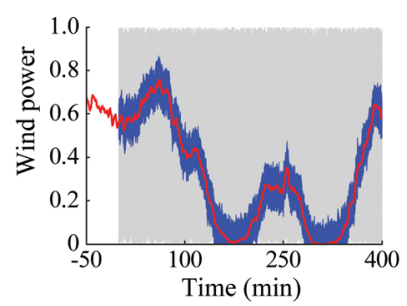

(a) $100 \%$ scenarios captured band

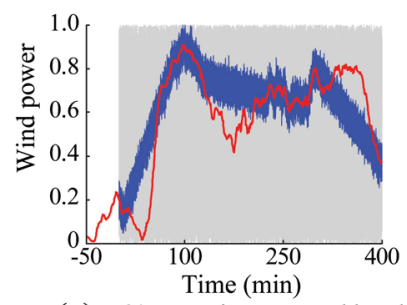

(c) $60 \%$ scenarios captured band

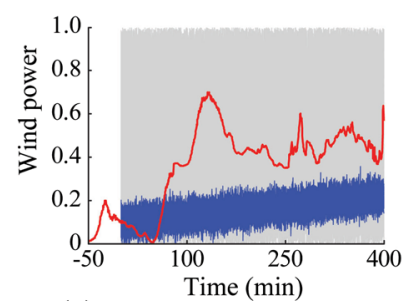

(e) $20 \%$ scenarios captured band

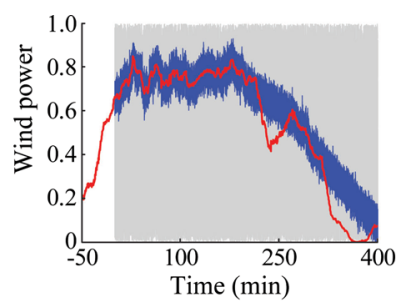

(b) $80 \%$ scenarios captured band

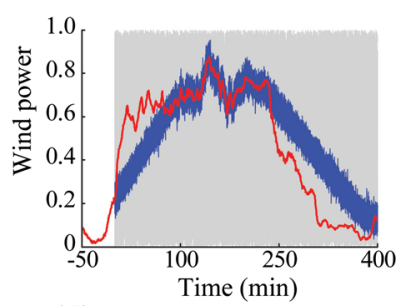

(d) $40 \%$ scenarios captured band

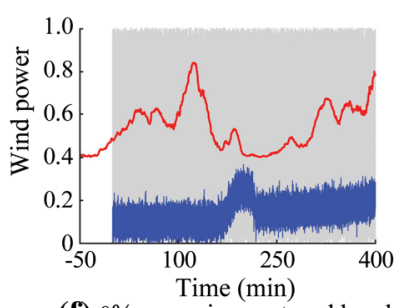

(f) $0 \%$ scenarios captured band
Fig. 8 Prediction results of different statistical scenarios in $50 \%$ confidence interval

Especially, the proportion of $100 \%$ SSCB partially equals to the corresponding confidence interval value. After the SSCB is formulated, the ramp event detecting algorithm can be fully used to extract the forecasted characteristics of ramp events.

\subsection{Independent distribution experiments of the single characteristic}

Detected ramp events in Fig. 5 and SSCBs in Fig. 7a are taken for example. Sevlian detecting algorithm is used to identify all the ramp events (up-ramps and down-ramps) and characteristics. The statistical analysis is made through a large amount of forecasted characteristic data. Methods for solving model parameters with the single objective function as the fitness function are compared. Statistical results of three ramp event characteristics are shown in Fig. 11 and Table 2. In Fig. 11, solid, dashed and dotted lines represent the multi-objective, $\mathrm{CDF}$ and secondary order moment autocorrelation fitness functions, respectively.

The corresponding maximum probability density value can be calculated from ramp characteristic statistics and is most likely to occur. Therefore, these statistical results are recorded in Table 2.
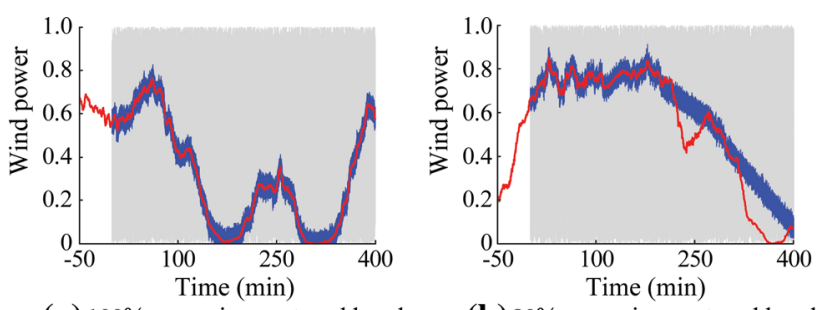

(a) $100 \%$ scenarios captured band

(b) $80 \%$ scenarios captured band

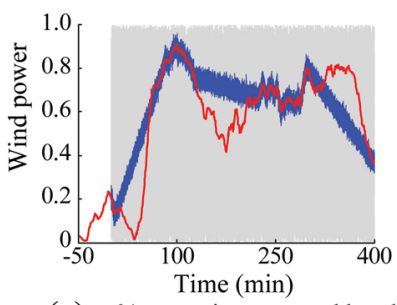

(c) $60 \%$ scenarios captured band

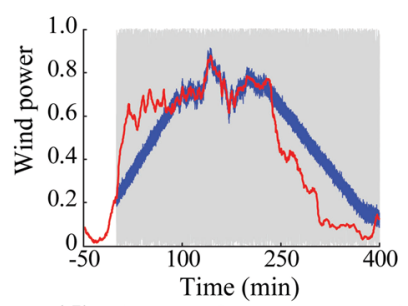

(d) $40 \%$ scenarios captured band

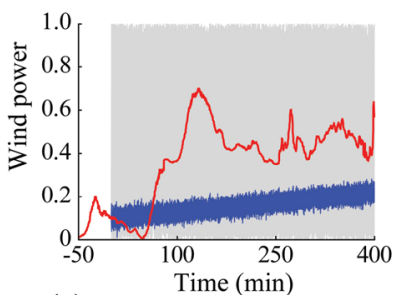

(e) $20 \%$ scenarios captured band

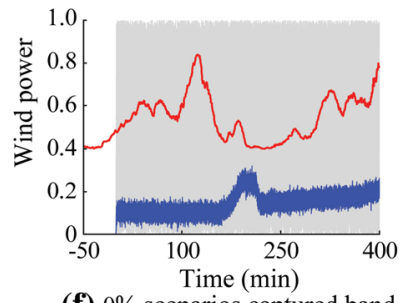

- Actural wind power; - Unsnapped scenarios; $-\mathrm{SSCBs}$

Fig. 9 Prediction results of different statistical scenarios in $30 \%$ confidence interval

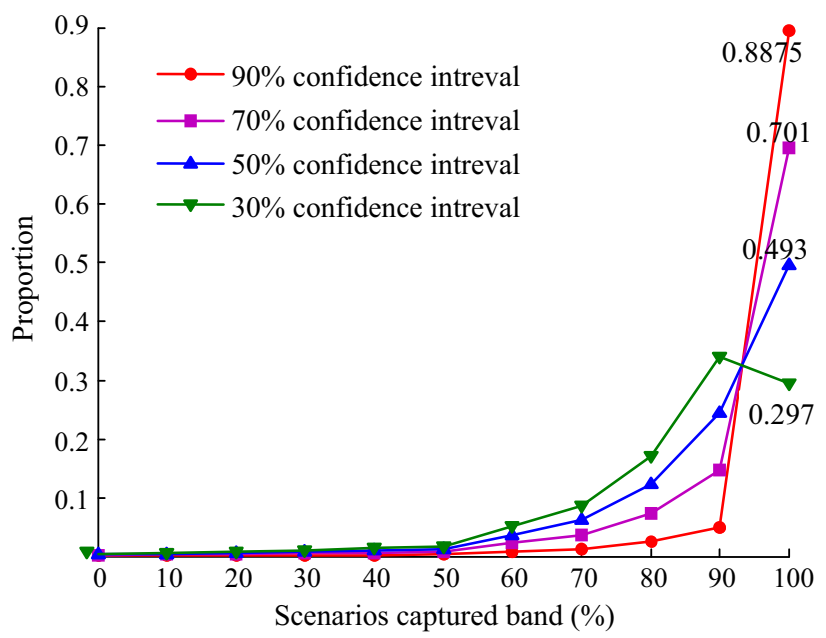

Fig. 10 Statistical proportion of the SSCBs

Figure 11 and Table 2 illustrate that the proposed method for the multi-objective fitness function is more accurate than the other two methods only using the CDF or secondary order moment autocorrelation (SecMo) fitness function separately. This is because the multi-objective fitness function considers not only the wind power distribution characteristics of historical wind power data but also 


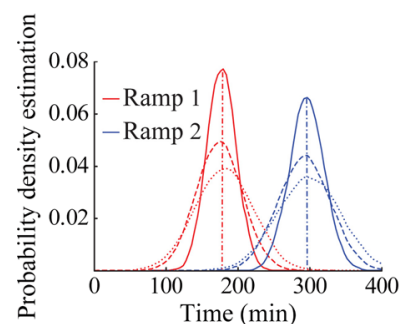

(a) Estimated ramp-ups start time

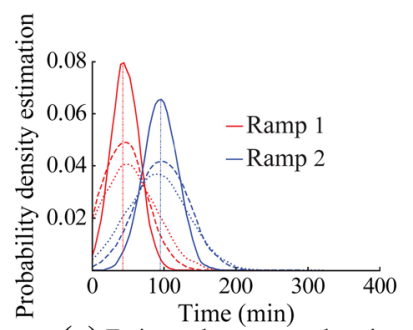

(c) Estimated ramp-ups duration

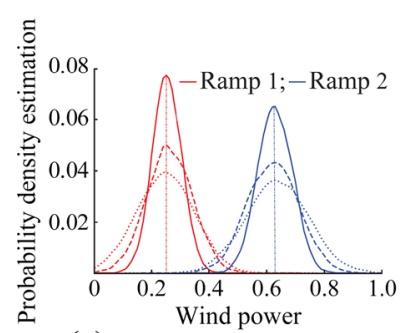

(e) Estimated ramp-ups swing

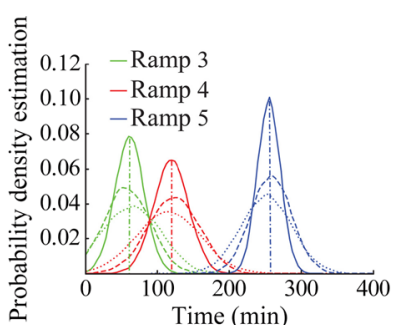

(b) Estimated ramp-downs start time

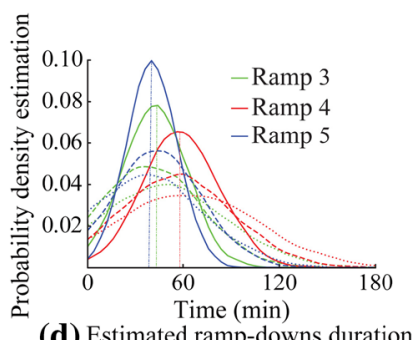

(d) Estimated ramp-downs duration

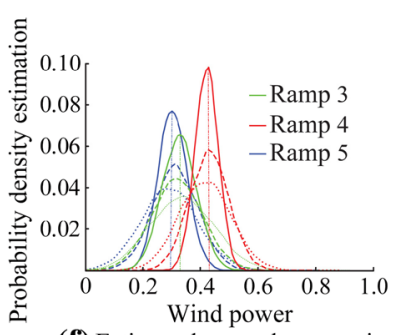

(f) Estimated ramp-downs swing

Fig. 11 Probability density estimation charts of the characteristics

the correlation degree between the predicted and actual wind power data. The reason that secondary order moment features are more important is that each characteristic of ramp events is closely related to the temporality of actual wind power data. During the iterative process of forecasting output data, secondary order moment features can make

sorting features of output data fully conform to that of actual wind power data and guarantee the strong correlations between them. It can be seen that probability density estimation values calculated by the three methods are different, which means that statistical results of multi-objective fitness functions are more concentrated and have statistical significance.

\subsection{Analysis of forecasting results on different SSCBs}

The evaluation of statistical scenarios forecasting models is based on the deviation between the forecasted and actual values of wind power ramp event characteristics rather than the pure wind power. In order to evaluate the robustness of the statistical scenarios forecasting model, two evaluation criteria are defined to characterize the error relationship between forecasted and actual values of ramp event characteristics. The normalized mean absolute error $\left(E_{\mathrm{NMAE}}\right)$ and the root mean square error $\left(E_{\mathrm{RMSE}}\right)$ criteria are defined as;

$E_{\mathrm{NMAE}}=\frac{1}{N_{\mathrm{S}}} \sum_{t=1}^{N_{\mathrm{S}}} \frac{\left|R_{\mathrm{P}}(t)-R_{\mathrm{PF}}(t)\right|}{P_{\mathrm{N}}} \times 100 \%$

$E_{\mathrm{RMSE}}=\sqrt{\frac{1}{N_{\mathrm{S}}} \sum_{t=1}^{N_{\mathrm{S}}}\left(R_{\mathrm{P}}(t)-R_{\mathrm{PF}}(t)\right)^{2}}$

where $R_{\mathrm{P}}(t)$ and $R_{\mathrm{PF}}(t)$ are actual and forecasted values of ramp event characteristics at time $t$, respectively; $P_{\mathrm{N}}$ is the nameplate capacity of a wind turbine or wind plant; $N_{\mathrm{S}}$ indicates the number of samples in the forecasting horizon. $70 \%$ confidence interval is taken and statistical results of forecasting error criteria are shown in Table 3.

Table 2 Statistical results of three ramp characteristics

\begin{tabular}{|c|c|c|c|c|c|c|}
\hline \multirow[t]{2}{*}{ Statistical results } & & \multicolumn{2}{|c|}{ Ramp-ups } & \multicolumn{3}{|c|}{ Ramp-downs } \\
\hline & & Ramp 1 & Ramp 2 & Ramp 3 & Ramp 4 & Ramp 5 \\
\hline \multirow[t]{4}{*}{ Ramp start time (min) } & Actual & 177 & 296 & 61 & 119 & 256 \\
\hline & Multi & 179 & 298 & 62 & 118 & 255 \\
\hline & $\mathrm{CDF}$ & 168 & 290 & 49 & 125 & 262 \\
\hline & SecMo & 188 & 305 & 70 & 107 & 251 \\
\hline \multirow[t]{4}{*}{ Ramp duration (min) } & Actual & 46 & 94 & 42 & 58 & 40 \\
\hline & Multi & 47 & 96 & 42 & 59 & 39 \\
\hline & $\mathrm{CDF}$ & 50 & 100 & 36 & 61 & 44 \\
\hline & SecMo & 53 & 84 & 49 & 68 & 36 \\
\hline \multirow[t]{4}{*}{ Ramp swing (p.u.) } & Actual & 0.2533 & 0.6281 & 0.3267 & 0.4216 & 0.3035 \\
\hline & Multi & 0.2538 & 0.6283 & 0.3265 & 0.4289 & 0.2956 \\
\hline & $\mathrm{CDF}$ & 0.2549 & 0.6289 & 0.3032 & 0.4292 & 0.3143 \\
\hline & SecMo & 0.2552 & 0.6293 & 0.3375 & 0.4101 & 0.2820 \\
\hline
\end{tabular}


Table 3 Forecasting error criteria statistics of different SSCBs

\begin{tabular}{|c|c|c|c|c|c|c|c|c|c|c|}
\hline \multirow{2}{*}{$\begin{array}{l}\text { SSCBs } \\
(\%)\end{array}$} & \multicolumn{2}{|c|}{ Ramp start time } & \multicolumn{2}{|c|}{ Ramp duration } & \multicolumn{2}{|c|}{ Ramp end time } & \multicolumn{2}{|c|}{ Ramp swing } & \multicolumn{2}{|c|}{ Ramp rate } \\
\hline & $\begin{array}{l}\text { NMAE } \\
(\%)\end{array}$ & $\begin{array}{l}\text { RMSE } \\
(\min )\end{array}$ & $\begin{array}{l}\text { NMAE } \\
(\%)\end{array}$ & $\begin{array}{l}\text { RMSE } \\
(\mathrm{min})\end{array}$ & $\begin{array}{l}\text { NMAE } \\
(\%)\end{array}$ & $\begin{array}{l}\text { RMSE } \\
(\mathrm{min})\end{array}$ & $\begin{array}{l}\text { MAE } \\
(\%)\end{array}$ & $\begin{array}{l}\text { RMSE } \\
\text { (p.u.) }\end{array}$ & $\begin{array}{l}\text { MAE } \\
(\%)\end{array}$ & $\begin{array}{l}\text { RMSE } \\
\text { (MW/min) }\end{array}$ \\
\hline 100 & 8.69 & 4.95 & 12.36 & 5.30 & 9.03 & 3.50 & 15.43 & 0.05 & 15.69 & 0.12 \\
\hline 90 & 20.17 & 5.36 & 18.35 & 6.96 & 11.72 & 5.54 & 25.39 & 0.05 & 22.36 & 0.26 \\
\hline 80 & 23.31 & 5.65 & 30.88 & 13.92 & 23.98 & 6.56 & 25.92 & 0.13 & 29.81 & 0.32 \\
\hline 70 & 23.65 & 6.55 & 40.40 & 14.50 & 36.37 & 10.91 & 43.39 & 0.16 & 33.12 & 0.48 \\
\hline 60 & 37.82 & 6.60 & 43.47 & 16.72 & 41.90 & 11.15 & 54.90 & 0.21 & 51.38 & 0.54 \\
\hline 50 & 40.34 & 9.02 & 48.14 & 17.69 & 51.41 & 12.42 & 68.42 & 0.27 & 66.11 & 0.54 \\
\hline 40 & 53.21 & 12.48 & 55.14 & 17.91 & 60.76 & 13.98 & 74.37 & 0.31 & 71.19 & 0.64 \\
\hline 30 & 58.97 & 12.64 & 55.30 & 21.58 & 70.57 & 16.91 & 80.00 & 0.36 & 74.60 & 0.65 \\
\hline 20 & 62.89 & 26.24 & 95.12 & 23.36 & 70.70 & 17.10 & 83.02 & 0.39 & 80.34 & 0.65 \\
\hline 10 & 84.83 & 26.78 & 95.38 & 24.92 & 78.74 & 18.70 & 83.46 & 0.41 & 87.89 & 0.87 \\
\hline 0 & 98.43 & 28.41 & 99.48 & 26.24 & 87.45 & 24.81 & 97.98 & 0.43 & 96.42 & 0.94 \\
\hline
\end{tabular}

Table 3 indicates the prediction effect of the 100\% SSCB prefers on other SSCBs with respect to ramp event characteristics. It can capture the variation trend of actual wind power with smaller errors in a set of confidence interval, which verifies the veracity of the $100 \%$ SSCB. Furthermore, the $100 \%$ SSCB occupies $70.12 \%$ of all the SSCBs, as shown in Fig. 10, which further verifies the robustness of the statistical scenarios forecasting model. It is worth nothing that although the 0\% SSCB cannot forecast wind power totally (due to the $0 \%$ probability), there is also a small probability to identify ramp event characteristics, which is the difference between wind power forecasting and ramp event forecasting.

\section{Conclusions}

In this paper, a new forecasting strategy for statistical wind power ramp events is proposed. The improved genetic algorithm based on multi-objective fitness functions is preformed to optimize parameters of the NN probability generating model in order to produce a number of statistical scenarios. After detecting ramp event characteristics in the statistical scenarios captured bands, the efficiency of the forecasting model can be evaluated by ramp features. Through the simulation, the calculation and the statistical analysis, conclusions are presented as follows:

1) When the confidence interval is greater than $50 \%$, the $100 \%$ SSCB cannot completely capture the wind power and occupy the highest proportion among all the SSCBs. This phenomenon verifies the robustness of the statistical scenarios forecasting model.

2) Multi-objective fitness functions can consider distribution and timing characteristics of the actual wind power. Results by using the method are more accurate than that by using single objective function.

3) Correlations of ramp-up characteristics are lower than that of ramp-down characteristics. Ramp duration canot exceed $150 \mathrm{~min}$ in the period of $400 \mathrm{~min}$ normally. Moreover along with the last ramp event occurring, the occurring possibility of the next ramp event is higher.

4) SSCBs are very robust for detecting ramp event characteristics and can be used as a method for forecasting wind power ramp events.

However, there are still some large errors in the SSCBs. In order to simplify the calculation process, only the secondary order moment as a representative of higher order moments and 6 inputs 4 hidden nodes as an assumption of more variables are used in the multi-objective fitness functions because of our computational conditions. Nevertheless, as a line of thinking of using higher order moment for wind power ramp even forecasting, the secondary order moment is still effective. In the next step work, some higher order moment, such as the third order moment, will be performed to improve the forecasting effects. Besides, more inputs and hidden nodes will be tested with larger computable servers, especially parallel computing.

Acknowledgments This work was supported by the National Basic Research Program of China (No. 2012CB215101). The authors would like to thank the anonymous reviewers for their constructive suggestions to this research.

Open Access This article is distributed under the terms of the Creative Commons Attribution 4.0 International License (http://creativecommons.org/licenses/by/4.0/), which permits unrestricted use, distribution, and reproduction in any medium, provided you give appropriate credit to the original author(s) and the source, provide a link to the Creative Commons license, and indicate if changes were made. 


\section{References}

[1] Sorensen P, Cutululis NA, Vigueras-Rodriguez A et al (2007) Power fluctuations from large wind farms. IEEE Trans Power Syst 22(3):958-965

[2] Zheng HY, Kusiak A (2009) Prediction of wind farm power ramp rates: a data-mining approach. J Solar Energy Eng 131(3):031011/1-8

[3] Navid N, Rosenwald G (2012) Market solutions for managing ramp flexibility with high penetration of renewable resource. IEEE Trans Sustain Energy 3(4):784-790

[4] AWS (2008) Truewind's final report for the Alberta forecasting pilot project. AWS Truewind, Albany

[5] Kamath C (2010) Understanding wind ramp events through analysis of historical data. In: Proceedings of the 2010 IEEE PES transmission and distribution conference and exposition, New Orleans, LA, USA, 19-22 Apr 2010, 6 pp

[6] Wang YF, Chen NM (2009) Impact of WRIG and DFIG wind generation ramping down on independent power systems. In: Proceedings of the 2009 IEEE PES/IAS conference on sustainable alternative energy (SAE'09), Valencia, Spain, 28-30 Sept 2009, 7 pp

[7] Kamath C (2011) Associating weather conditions with ramp events in wind power generation. In: Proceedings of the 2011 IEEE PES power systems conference and exposition (PSCE'11), Phoenix, AZ, USA, 20-23 Mar 2011, 8 pp

[8] Freedman J, Markus M, Penc R (2008) Analysis of West Texas wind plant ramp-up and ramp-down events. AWS Truewind, Albany

[9] Ferreira C, Gama J, Matias L et al (2010) A survey on wind power ramp forecasting. ANL/DIS-10-13, Argonne National Laboratory (ANL), Cass Avenue Argonne, IL, USA

[10] Barber C, Bockhorst J, Roebber P et al (2010) Auto-regressive HMM inference with incomplete data for short-horizon wind forecasting. In: Proceedings of the 24th annual conference on neural information processing systems (NIP'10), Vancouver, Canada, 6-11 Dec 2010, 9 pp

[11] Gallego C, Costa A, Cuerva A (2010) Improving short-term forecasting during ramp events by means of regime-switching artificial neural networks. In: Proceedings of the 10th EMS annual meeting and 8th European conference on applied climatology (ECAC'10), Zurich, Switzerland, 13-17 Sept 2010, pp 55-58

[12] Zareipour H, Huang DL, Rosehart W (2011) Wind power ramp events classification and forecasting: A data mining approach. In: Proceedings of the 2011 IEEE Power and Energy Society general meeting, San Diego, CA, USA, 24-29 Jul 2011, 3 pp

[13] Bradford KT, Carpenter RL, Shaw BL (2010) Forecasting southern plains wind ramp events using the WRF model at 3-km. In: Proceedings of the 9th annual American Meteorological Society (AMS) student conference, 17 Jan 2010, 10 pp

[14] Sevlian R, Rajagopal R (2013) Detection and statistics of wind power ramps. IEEE Trans Power Syst 28(4):3610-3620

[15] Cutler N, Kay M, Jacka K et al (2007) Detecting categorizing and forecasting large ramps in wind farm power output using meteorological observations and WPPT. Wind Energy $10(5): 453-470$
[16] Bossavy A, Girard R, Kariniotakis G (2010) Forecasting uncertainty related to ramps of wind power production. In: Proceedings of the European wind energy conference and exhitition (EWEC'10), Warsaw, Poland, 20-23 Apr 2010, 9 pp

[17] Greaves B, Collins J, Parkes J et al (2009) Temporal forecast uncertainty for ramp events. Wind Eng 33(4):309-320

[18] Sexauer JM (2012) Development of probabilistic load flow for voltage quality analysis in the presence of distributed generation. Ph.D. Thesis, Colorado School of Mines, Golden, CO, USA

[19] Cardoso JF (1989) Source separation using higher order moments. In: Proceedings of the 1989 international conference on acoustics, speech, and signal processing (ICASSP'89), vol 4, Glasgow, UK, 23-26 May 1989, pp 2109-2112

[20] Giebel G, Brownsword R, Kariniotakis G et al (2011) The stateof-the-art in short-term prediction of wind power: a literature overview, 2nd edn. ANEMOS plus, Roskilde

[21] Pinson P, Madsen H, Nielsen HA et al (2009) From probabilistic forecasts to statistical scenarios of short-term wind power production. Wind Energy 12(1):51-62

[22] Sevlian R, Rajagopal R (2012) Wind power ramps: detection and statistics. In: Proceedings of the 2012 IEEE Power and Energy Society general meeting, San Diego, CA, USA, 22-26 Jul 2012, 8 pp

23. Sevlian R, Rajagopal R (2012) Software for sliding window ramp detection. http://www.stanford.edu/ rsevlian/WindRampDetect. html. Accessed May 2012

Mingjian CUI received the B.E. degree in electrical engineering and automation from Wuhan University, Wuhan, China, in 2010, where he is currently working toward the Ph.D degree in electrical engineering. $\mathrm{He}$ is also a Visiting Scholar with the Transmission and Grid Integration Group (TGIG), National Renewable Energy Laboratory (NREL), Golden, CO, USA. His current research interests include wind power ramp events forecasting and power system dynamics and control.

Deping KE received the B.S. and M.S. degree in electrical engineering from Huazhong University of Science and Technology, Wuhan, China, in 2005 and 2007, respectively, and the Ph.D degree in electrical engineering from Hong Kong Polytechnic University, Hong Kong, China, in 2012.

Di GAN received the B.E. degree in electrical engineering and automation from Wuhan University, Wuhan, China, in 2013, where he is currently working toward the Master's degree in electrical engineering. His current research interest includes wind power forecasting.

Yuanzhang SUN received the B.S. degree from Wuhan University of Hydro and Electrical Engineering, Wuhan, China, in 1987, the M.S. degree from the Electric Power Research Institute (EPRI), Beijing, China, in 1982, and the Ph.D degree in electrical engineering from Tsinghua University, Beijing, in 1988. 